The Astrophysical Journal, 489:87-93, 1997 November 1

(C) 1997. The American Astronomical Society. All rights reserved. Printed in U.S.A.

\title{
EVIDENCE FOR A PRECESSING ACCRETION DISK IN THE NUCLEUS OF NGC 1097 \\ Thaisa Storchi-Bergmann, ${ }^{1}$ Michael Eracleous, ${ }^{2,3}$ María Teresa Ruiz, ${ }^{4,5}$ Mario Livio, ${ }^{6}$ \\ ANDREW S. WILSON, ${ }^{7}$ AND ALEXEI V. FiliPPENKo ${ }^{3}$ \\ Received March 13, 1997; accepted 1997 June 9
}

\begin{abstract}
We present new spectroscopic observations of the LINER (and now Seyfert 1) nucleus of NGC 1097, and discuss the evolution of its broad, double-peaked Balmer lines. When originally discovered in 1991, the red peak of the double-peaked $\mathrm{H} \alpha$ line was stronger than the blue, while by 1994 the $\mathrm{H} \alpha$ profile had become almost symmetric and the integrated line flux had decreased to half its original value. Our new spectrum, taken in 1996, shows that the broad, double-peaked lines have returned to almost their original strengths, the profiles of $\mathrm{H} \beta$ and $\mathrm{H} \alpha$ are identical to within errors, and the broad-line emitting region is unreddened. However, the profile of the Balmer lines is now such that the blue peak is stronger than the red, opposite to the asymmetry observed in 1991. Various models are considered for the observed behavior, all assuming that the emission lines originate in an accretion disk. We present a refined version of the precessing, planar, elliptical accretion-ring model proposed by Storchi-Bergmann et al. and Eracleous et al. This model provides an acceptable fit to the line profiles. We also consider the possibility that the line-profile evolution results from a precessing warp in the disk, induced by irradiation from the center, and show that the range of radii and precession timescales expected in this model are consistent with the observations. The sudden appearance of the "disklike" broad-line profiles in NGC 1097 could have resulted from the formation of a new accretion disk due, for example, to the tidal disruption of a star or the illumination of a preexisting disk by a transient ionizing source at the center of the disk.
\end{abstract}

Subject headings: accretion, accretion disks — galaxies: individual (NGC 1097) - galaxies: nuclei galaxies: Seyfert - line: profiles

\section{INTRODUCTION}

In the most widely accepted picture for active galactic nuclei (AGNs), a nuclear, supermassive black hole is fed by an accretion disk. Nevertheless, observational evidence for the presence of the disk has been limited and indirect until very recently (see, for example, Koratkar, Kinney, \& Bohlin 1992; Koratkar et al. 1995; Antonucci 1992; Antonucci, Kinney, \& Ford 1989). There was no clear, dynamical signature of the disk in the form of double-peaked emission lines, such as those found in cataclysmic variable stars (e.g., Young \& Schneider 1980; Young, Schneider, \& Shectman 1981; Horne \& Marsh 1986; Marsh 1988). It is now known that the X-ray spectra of most Seyfert 1 galaxies possess broad, disk-like iron $\mathrm{K} \alpha$ lines (Tanaka et al. 1995; Mushotzky et al. 1995; Nandra et al. 1997). Moreover, about $10 \%$ of broad-line radio galaxies show disk-like Balmer lines with twin peaks or twin shoulders (Eracleous \& Halpern 1994, hereafter EH94). These disklike Balmerline profiles are approximately twice as broad as the "normal" broad lines, and they are very well reproduced by models of gas rotating at relativistic speeds in a Keplerian

\footnotetext{
${ }^{1}$ Instituto de Física, UFRGS, CP 15051, Porto Alegre, RS, Brazil; thaisa@if.ufrgs.br.

${ }^{2}$ Hubble Fellow.

${ }^{3}$ Department of Astronomy, University of California, Berkeley, CA 94720-3411; mce@beast.berkeley.edu, alex@wormhole.berkeley.edu.

${ }^{4}$ Universidad de Chile, Casilla 36-D, Santiago, Chile; mtruiz@das.uchile.cl.

${ }^{5}$ Visiting Astronomer, Cerro Tololo Inter-American Observatory. CTIO is operated by AURA, Inc. under contract to the National Science Foundation.

${ }^{6}$ Space Telescope Science Institute, 3700 San Martin Drive, Baltimore, MD 21218; mlivio@stsci.edu.

${ }_{7}$ Astronomy Department, University of Maryland, College Park, MD 20742; wilson@astro.umd.edu.
}

accretion disk. The relative strengths of the narrow lines of these radio-loud "disklike" emitters are similar to those found in low-ionization nuclear emission-line regions (LINERs; Heckman 1980).

The association of broad, double-peaked Balmer lines (whatever their origin) with objects displaying LINER-like narrow lines has been underscored by the recent abrupt appearance of double-peaked Balmer lines in three LINERs and LINER-like objects that did not previously have them: NGC 1097 (Storchi-Bergmann, Baldwin, \& Wilson 1993), Pictor A (Halpern \& Eracleous 1994), and M81 (Bower et al. 1996). While Pictor A is a radio galaxy with similar characteristics to those of the radio-loud "disklike" emitters found by EH94, NGC 1097 and M81 are spiral galaxies with low-luminosity LINER nuclei and much weaker radio emission. Nevertheless, NGC 1097 and M81 show similarities to the more luminous double-peaked emitters found by EH94, as both have nuclear nonthermal radio sources with a synchrotron self-absorbed spectrum, characteristic of compact jets (Hummel, van der Hulst, \& Keel 1987; Bartel et al. 1982). Moreover, NGC 1097 is famous for its optical "jets" (Wolstencroft \& Zealey 1975), while the nucleus of M81 harbors an elongated radio structure found by VLBI mapping (Bietenholz et al. 1996). The nuclei of NGC 1097 and M81 are also hard X-ray sources (Iyomoto et al. 1996; Ishisaki et al. 1996).

In the case of NGC 1097, Eracleous et al. (1995) were able to reproduce the shape of the double-peaked profile with a model of an elliptical accretion ring. An elliptical, rather than circular, ring was required in order to reproduce a double-peaked line with the red peak stronger than the blue one. In a subsequent paper, Storchi-Bergmann et al. (1995) presented the results of monitoring the NGC $1097 \mathrm{H} \alpha$ profile: after approximately 2 years, the profile had become 


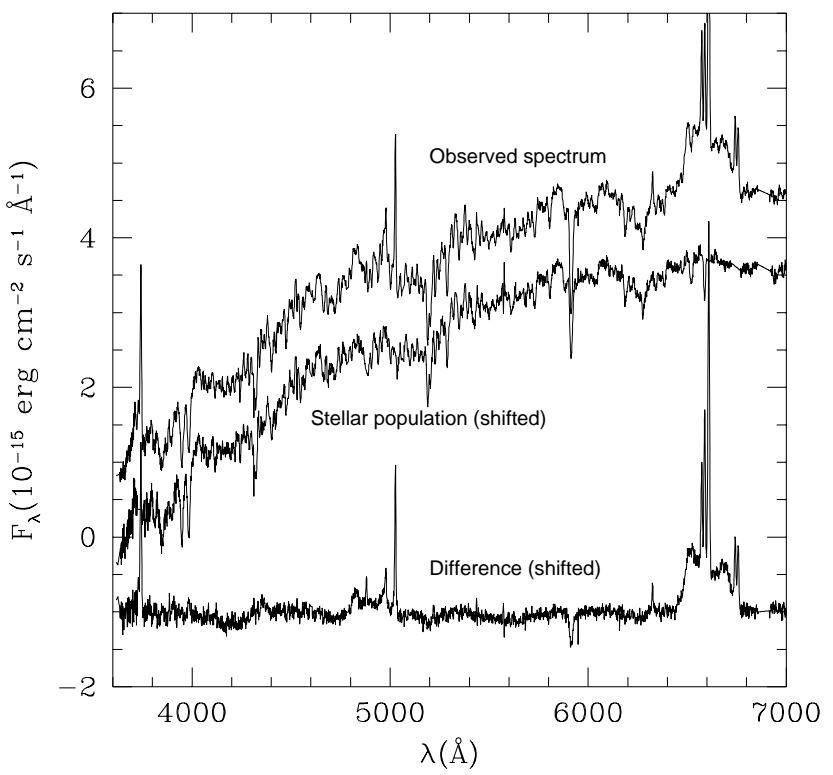

FIG. 1.-Observed nuclear spectrum (top), the adopted stellar population spectrum (middle), and the difference between the two (bottom), within a $2^{\prime \prime} \times 4$ " aperture. The two last spectra have been shifted for clarity.

more symmetric, with the two peaks showing similar heights. This change was interpreted in the context of the elliptical accretion ring model as a result of relativistic precession of the ring (advance of the pericenter), which yielded an estimate of the black hole mass of about $10^{6} M_{\odot}$. Here we present a new spectrum showing that the profile has continued to vary, in a manner consistent with an origin in a precessing accretion disk. We do, however, find it neces-

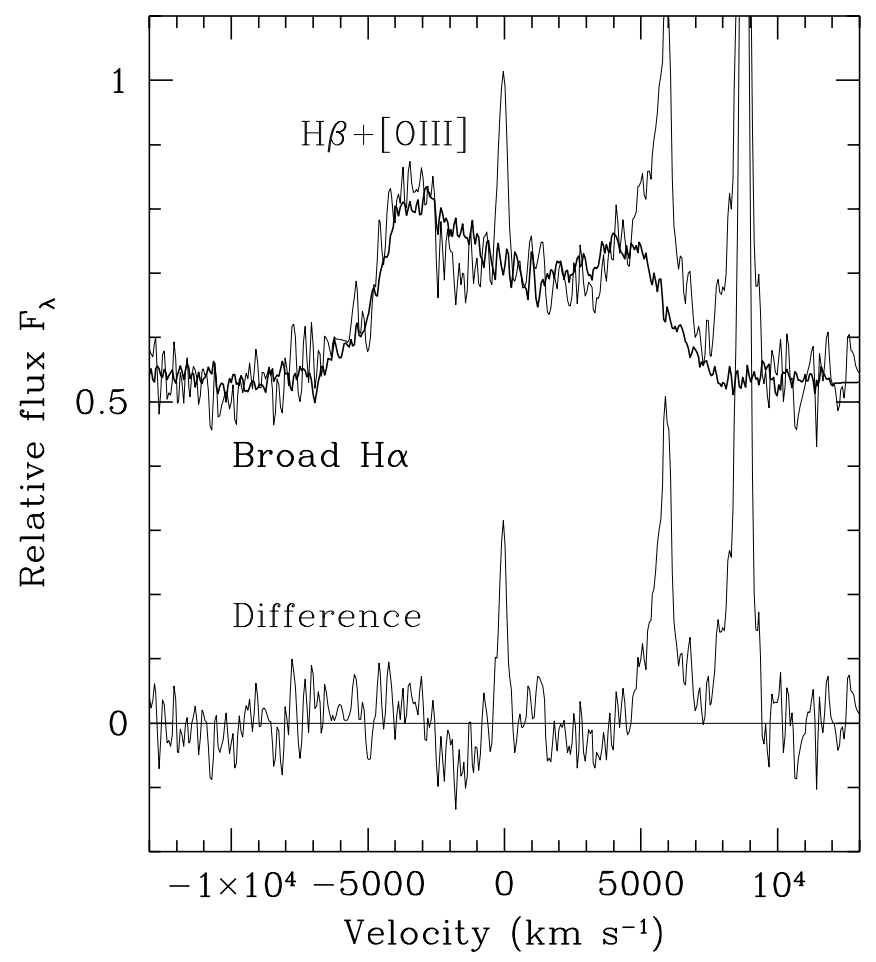

Fig. 2.-Top: Comparison between the double-peaked $\mathrm{H} \alpha$ profile (heavy line), divided by 2.8 (see text), and the $\mathrm{H} \beta+[\mathrm{O}$ III $] \lambda \lambda 4959,5007$ profiles in velocity space. Bottom: The difference $(\mathrm{H} \beta+[\mathrm{O} \mathrm{III}])-(\mathrm{H} \alpha / 2.8)$. sary to refine the original model of Storchi-Bergmann et al. (1995) so that it can account self-consistently for all of the available data.

\section{OBSERVATIONS AND RESULTS}

A new long-slit optical spectrum of the NGC 1097 nucleus was obtained using the Cassegrain spectrograph and Loral $3 \mathrm{~K} \mathrm{CCD}$ detector at the $4 \mathrm{~m}$ telescope of the Cerro Tololo Inter-American Observatory on 1996 January 24 UT. The spectral range covered was 3500-7000 $\AA$, at $4 \AA$ resolution. During the observation, the $2^{\prime \prime}$ slit was oriented at position angle $83^{\circ}$. The zenith angle was small, and therefore the effects of atmospheric dispersion were negligible. The spectrum was reduced and calibrated using standard procedures and with the help of the IRAF software package. In order to isolate the emission from the gas, we subtracted the contribution of the stellar population, which was obtained by averaging two spectra extracted from approximately $5^{\prime \prime}$ east and $5^{\prime \prime}$ west of the nucleus. To match the slope of the off-nuclear spectrum to the starlight spectrum of the nucleus, it was necessary to redden the former by $E(B-V)=0.12 \mathrm{mag}$ using a Galactic reddening law (Seaton 1979).

Figure 1 shows the observed nuclear spectrum, together with the starlight spectrum and the difference between the two. In the starlight-subtracted spectrum, the doublepeaked profile can be readily seen, not only in $\mathrm{H} \alpha$ but also in $\mathrm{H} \beta$. The most striking feature in the difference spectrum is the shape of the double-peaked profiles: the blue peak is now stronger than the red, the opposite asymmetry to that of the first observations of 1991 (Storchi-Bergmann et al. 1993). The flux in the double-peaked lines is $1.61 \pm 0.02 \times 10^{-13} \mathrm{erg} \mathrm{cm}^{-2} \mathrm{~s}^{-1}$ for $\mathrm{H} \alpha$ and $0.57 \pm 0.02 \times 10^{-13} \mathrm{erg} \mathrm{cm}^{-2} \mathrm{~s}^{-1}$ for $\mathrm{H} \beta$. In the difference spectrum, the usual narrow lines are evident, as well as the residual Na I D $5892 \AA$ interstellar absorption line, just as in the spectra of Storchi-Bergmann et al. (1995). It may be argued that a hint of broad $\mathrm{Fe}$ II emission in the spectral regions around 4570 and $5300 \AA$ can also be seen, but a deeper exposure would be necessary in order to confirm the presence of these features.

We show in Figure 2 a comparison of the $\mathrm{H} \alpha$ and $\mathrm{H} \beta$ profiles superposed in velocity space. The broad $\mathrm{H} \alpha$ profile was isolated by fitting and subtracting the narrow $\mathrm{H} \alpha$, [N II] $\lambda \lambda 6548,6584$, and [S II] $\lambda \lambda 6717,6731$ lines and then dividing by 2.8 , which is the observed ratio between the integrated fluxes of the broad $\mathrm{H} \alpha$ and $\mathrm{H} \beta$ lines. We conclude that, within the observational uncertainties, the two profiles match each other very well. This is confirmed by the result of subtracting the scaled broad $\mathrm{H} \alpha$ from the $\mathrm{H} \beta+[\mathrm{O} \mathrm{III}]$ complex (Fig. 2, bottom plot), which includes only the narrow emission lines and residuals comparable to the noise in the difference spectrum. This result shows that in 1996 January there was neither reddening of the region producing the double-peaked profiles nor differential reddening across the profile.

\section{DISCUSSION}

\subsection{Comparison with Previous Observations}

In Figure 3 we show the $\mathrm{H} \alpha$ profile of the last observation together with the two earlier spectra having similar spectral resolution and signal-to-noise ratio. At the bottom of Figure 3, we have plotted the $\mathrm{H} \alpha$ profile of 1991 November 


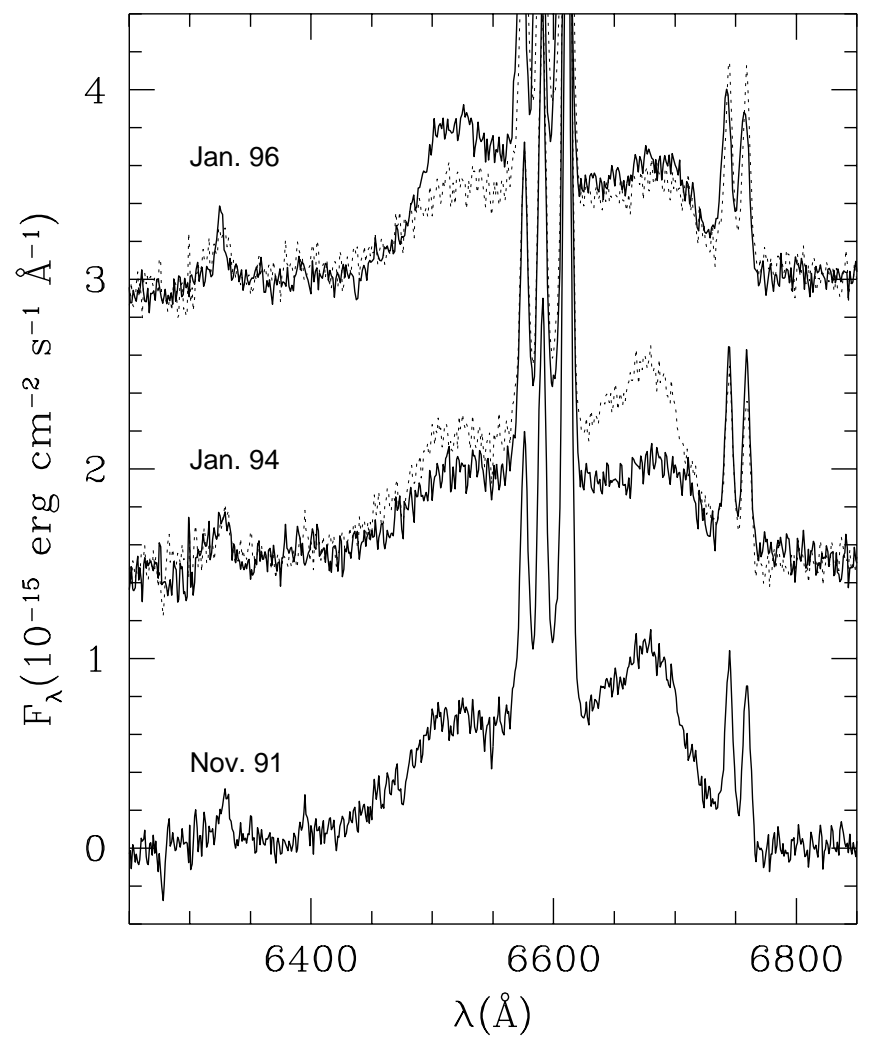

FIG. 3.-Double-peaked H $\alpha$ profiles in three distinct epochs. Dashed lines show the profile from the previous epoch, for comparison.

2 ; in the middle, that of 1994 January 4 ; and at the top, that of 1996 January 24 . These spectra, spaced by approximately 2 years, represent three characteristic snapshots in the continuous evolution of the $\mathrm{H} \alpha$ profile over the course of our observations.

From the line fluxes measured in the starlight-subtracted 1996 spectrum, analysis of the profiles in Figure 3, and the previous measurements presented in Storchi-Bergmann et al. (1995), we conclude the following:

1. The integrated flux in the broad $\mathrm{H} \alpha$ emission line, which decreased by $50 \%$ from 1991 November to 1994 January, has increased to $83 \%$ of its original flux.

2. The two peaks have kept the same velocities, $-3300 \pm 200 \mathrm{~km} \mathrm{~s}^{-1}$ for the blue peak and $3900 \pm 200 \mathrm{~km}$ $\mathrm{s}^{-1}$ for the red, relative to the narrow component of $\mathrm{H} \alpha$. This implies that the $\mathrm{H} \alpha$ line as a whole has not shifted appreciably. Thus, the average velocity of the two peaks relative to the narrow $\mathrm{H} \alpha$ is still $300 \pm 50 \mathrm{~km} \mathrm{~s}^{-1}$. We note that the heliocentric velocity of the narrow emission lines is consistent, within measurement uncertainties, with the heliocentric velocity derived from the stellar absorption lines (the two velocities differ by less than $40 \mathrm{~km} \mathrm{~s}^{-1}$ ).

3. The 1996 January profile of $\mathrm{H} \alpha$ is almost a mirror image of the 1991 November profile.

4. The detectable limit of the red wing relative to the narrow component of $\mathrm{H} \alpha$ is $8400 \pm 500 \mathrm{~km} \mathrm{~s}^{-1}$, and that of the blue wing is $-7200 \pm 500 \mathrm{~km} \mathrm{~s}^{-1}$, except in 1991 November, when the blue wing was more extended to about $-12,500 \pm 1000 \mathrm{~km} \mathrm{~s}^{-1}$.

5. The $\mathrm{H} \alpha / \mathrm{H} \beta$ ratio, which increased from $3.2 \pm 0.5$ in 1991 November to $4.2 \pm 0.7$ in 1993 September, has now decreased to $2.8 \pm 0.3$.
In Figure 4 we show some of the above conclusions graphically by plotting the variation of the broad $\mathrm{H} \alpha$ flux and the $\mathrm{H} \alpha / \mathrm{H} \beta$ ratio over the course of our observations.

\subsection{Line Emission from an Eccentric Accretion Ring}

The new spectrum of NGC 1097 presented here shows that the strength of the broad, double-peaked Balmer lines has recovered from the decline it was undergoing in 1994. The line profiles have been changing continuously since the first detection of the broad lines in 1991 November (see Storchi-Bergmann et al. 1993, 1995). The red peak of $\mathrm{H} \alpha$, which was initially stronger than the blue peak, has been steadily declining in strength. In the most recent spectrum, the blue peak has become stronger than the red one (see Figs. 1 and 3). This suggests very strongly that the observed line-profile variations are the result of a continuous process, which seems to operate independently of the variations in the integrated flux of the Balmer lines.

Storchi-Bergmann et al. (1995) considered a number of possible scenarios to account for the profiles of the doublepeaked Balmer lines and their variability, favoring in the end a model of an eccentric accretion ring (Eracleous et al. 1995). Such an eccentric ring is expected to result from the tidal disruption of a star by a supermassive black hole in the galactic nucleus (e.g., Rees 1988). The tidal disruption hypothesis is appealing because it can explain the abrupt appearance of the broad Balmer lines, and also because the formation and subsequent precession of an eccentric disk can explain the profiles of the broad lines and their variability. Storchi-Bergmann et al. (1995) also argued that the

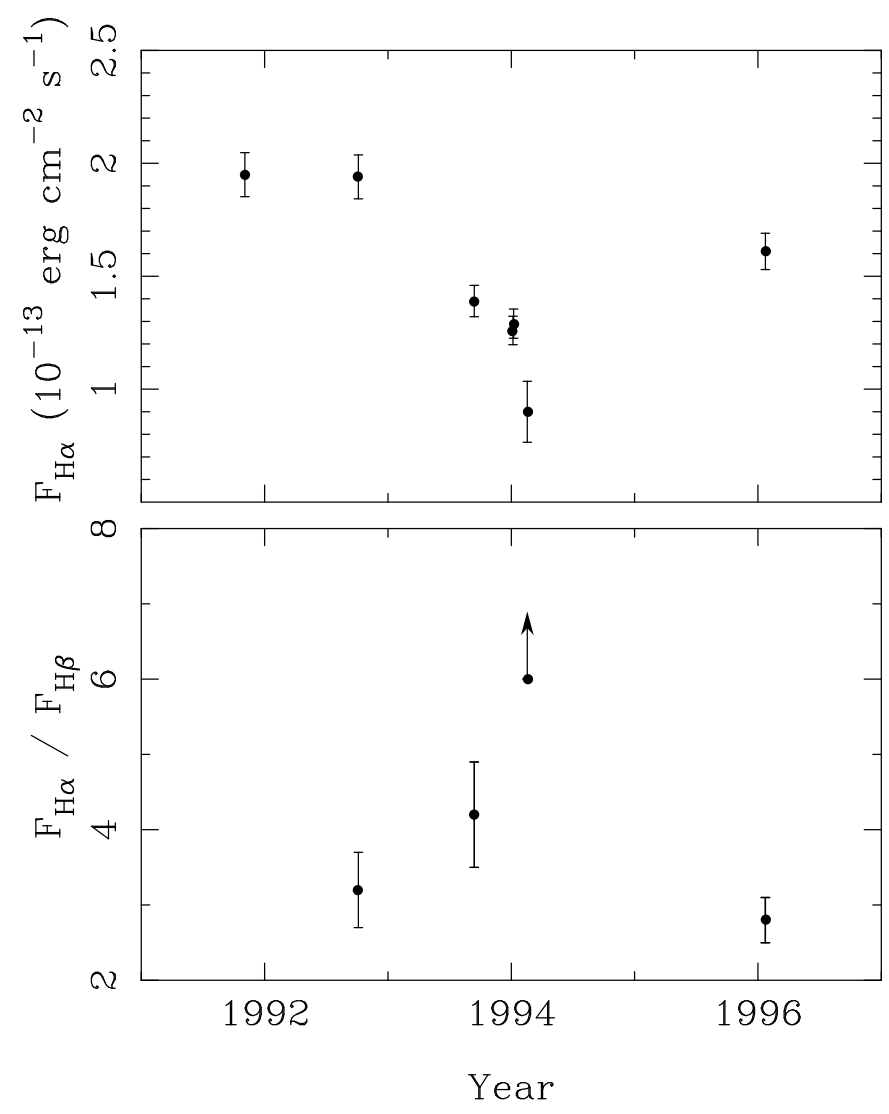

FIG. 4.-Variation of the broad $\mathrm{H} \alpha$ flux (top) and the $\mathrm{H} \alpha / \mathrm{H} \beta$ ratio (bottom) over the course of the NGC 1097 observations. 
variation of the profiles of the broad lines and the variation of the integrated line fluxes result from independent processes, which is supported by the new spectrum. Two plausible explanations were put forward for the decline of the integrated flux of the broad Balmer lines: $(a)$ the flux of ionizing radiation was itself declining, presumably as a result of a decreasing accretion rate, and $(b)$ an obscuring cloud was crossing the line of sight to the line-emitting ring (this cloud could be part of the interstellar medium in the immediate vicinity of the nucleus or a portion of the postdisruption debris that was able to expand adiabatically and reach a large height out of the plane of its original trajectory). Either of these hypotheses could account for the steepening of the Balmer decrement that accompanied the decline in the broad-line flux (see Fig. 4), but it was predicted that if the latter hypothesis is true, the broad lines should recover after the obscuring material left the line of sight. This is indeed what the new data show.

Not only were the elliptical-ring model for the line profile and the tidal disruption scenario for the appearance of the lines successful in explaining the observations, but their prediction for the evolution of the profile has now received strong support. In addition to accretion disk emission, two alternative scenarios are considered for the origin of broad, double-peaked emission lines: the bipolar outflow scenario (Zheng, Binette, \& Sulentic 1990) and the binary black hole hypothesis (Gaskell 1983). Independently of the variability properties of the line profiles, the binary black hole hypothesis appears unlikely because it requires that the supermassive binary in NGC 1097 would have to be of an unlikely type. The separation of the two peaks in the Balmer-line profiles implies that the two black holes should have roughly equal masses. If such a binary is to form from the merger of two parent galaxies, then the two parents should also have roughly equal masses. In all cases where the masses of black holes in the centers of active and nonactive galaxies have been determined by stellar or gas kinematics, the mass of the black hole is approximately proportional to the mass of the bulge of the parent galaxy (Kormendy et al. 1997). Therefore, the result of the merger would not be a spiral galaxy like NGC 1097. Models in which the double-peaked lines originate in the oppositely directed parts of a bipolar outflow are purely kinematic and thus notoriously difficult to constrain. These models can possibly accommodate the observed profile variability, although they cannot explain it in terms of a specific physical mechanism. Therefore, we do not consider these scenarios further and concentrate instead on accretion disk models. In the remainder of this section, we consider the eccentric-ring model in detail, while in the next section we discuss an alternative phenomenon associated with an accretion disk which may produce a similar behavior of the line profiles.

The specific model proposed by Storchi-Bergmann et al. (1995) involved an eccentric ring with a mean pericenter distance of $2600 r_{g}$ (where $r_{g} \equiv G M / c^{2}$ is the gravitational radius, with $M$ the mass of the black hole; $G$ is the gravitational constant; and $c$ is the speed of light), and a constant eccentricity of 0.5 . In this scenario, the precession of the ring, resulting from the general relativistic advance of the pericenter, was responsible for the smooth variation of the $\mathrm{H} \alpha$ line profile. The corresponding model profiles were in qualitative agreement with the observations. The inferred precession rate led to an estimate of the mass of the central black hole of approximately $10^{6} M_{\odot}$. We find it necessary to revise this model somewhat for two reasons. First, the direction of precession of the ring that was used to compute the model profiles was the opposite of that which general relativistic advance of the pericenter would produce, and was thus physically incorrect. The correct direction of precession produces a change in the line profiles in the opposite sense to that observed. Second, regardless of this error, a model involving an elliptical ring with a constant eccentricity is not able to fit all of the observed spectra in detail: the new spectrum presented here poses the most severe difficulty.

The most important revision that we propose is to make the eccentricity of the ring a function of radius (more precisely, of pericenter distance). We construct a model in which the inner annulus of the ring is circular and the eccentricity grows linearly with radius up to a maximum value of 0.45 . The plane of the ring is inclined at $34^{\circ}$ to the line of sight, and the line-emitting part of the ring is bounded by annuli with pericenter distances of $1300 r_{g}$ and $1600 r_{g}$. The ring is circular up to $1400 r_{g}$. At this radius it develops an eccentricity which increases linearly to its maximum value at the outer radius. An eccentricity that increases with radius is motivated by the results of the simulations of Syer \& Clarke (1992), which follow the evolution of the postdisruption debris from the tidal encounter of a star with a black hole. When the debris settles into a well-formed disk after about a viscous time, the inner parts have circularized as a result of differential precession, while the eccentricity of the outer particle orbits increases approximately linearly with radius. The model we are considering is purely kinematic, and it uses a parametric description of the accretion flow. As such, it may not conform well to the results of detailed hydrodynamical calculations. However, it allows us to draw conclusions about the general kinematic properties of the line-emitting gas. The modification that we have introduced adds one more free parameter to the disk model discussed by Storchi-Bergmann et al. (1995), which involved seven free parameters. One of the features of the refined model is that the predicted velocity shifts of the twin peaks resulting from the precession of the ring are small. This is a result of the combination of the steep emissivity law and the low eccentricity of the inner annuli of the disk where the emission is most intense.

In Figure 5 we show the detailed fits of the refined elliptical-ring model to the observed line profiles. We have chosen the three high-resolution spectra from our collection as examples; these also represent the beginning, middle, and end of the time sequence that we have observed so far. In the sequence of models presented here, we have allowed only for a precession of the elliptical ring, holding all other parameters fixed. In the 4.2 years covered by the spectra of Figure 5, the elliptical ring precesses by $60^{\circ}$ (i.e., the azimuthal angle of the apocenter increases smoothly from $320^{\circ}$ to $20^{\circ}$ ), which implies a precession period of 25 years and in turn a central black hole mass of $9 \times 10^{5} M_{\odot}$. We have arrived at this model by trial and error, judging the goodness of the fit by eye. At each individual epoch the bestfitting model is not unique. For example, the $\mathrm{H} \alpha$ model in 1991 November can be fitted equally well by the model presented by Eracleous et al. (1995) and by the model presented here. However, the information on the evolution of the profile with time restricts the possible models considerably. The inclination and the inner and outer radii of the 

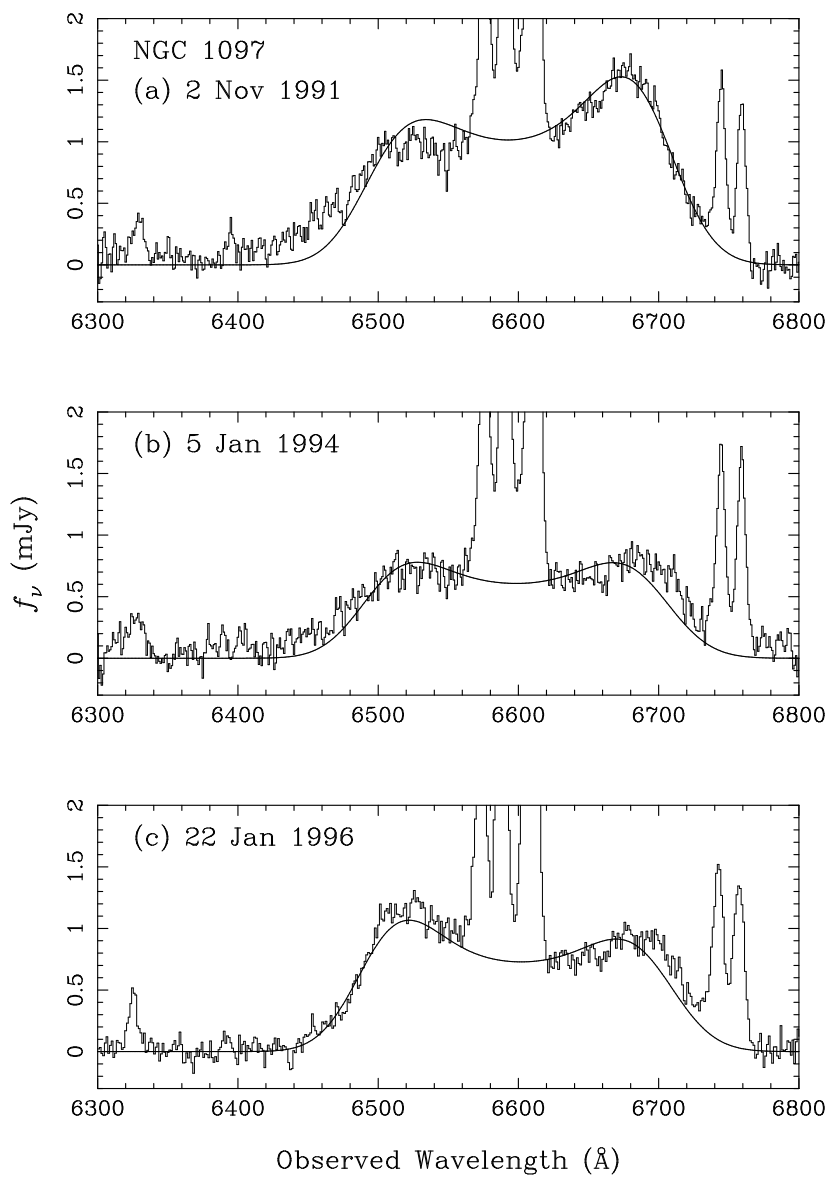

FIG. 5.-Detailed fits of the elliptical-ring model to the three highresolution $\mathrm{H} \alpha$ profiles of NGC 1097. In all cases, the continuum has been subtracted using an off-nuclear starlight spectrum (see text for details). The line-emitting ring has a circular inner annulus, followed by elliptical outer annuli whose eccentricity increases linearly outward up to a maximum value. The normal to the plane of the ring is inclined by $34^{\circ}$ to the line of sight. The inner circular annulus spans the radial range between $1300 r_{g}$ and $1400 r_{g}$, and the pericenter distance of the outer annulus is $1600 r_{g}$. The maximum (outer) eccentricity is 0.45 . In (a) the observer is located at an azimuth of $320^{\circ}$ (see definition in Eracleous et al. 1995). In (b) and (c) the ring is allowed to precess in steps of $30^{\circ}$.

ring can be constrained fairly well (within $10 \%$ ) by fitting the wings of the line profile. With these parameters held fixed, the orientation and eccentricity of the disk can be determined (to a similar uncertainty) by the requirement that the evolution of the profile is reproduced.

\subsection{Line Emission from a Precessing, Warped Disk}

The eccentric-ring scenario discussed above is successful in accounting for all of the observed features of the Balmer lines, including their profiles and their variability. It is still worthwhile, however, to consider alternative phenomena in the disk which could produce (qualitatively at least) a similar behavior. More specifically, we discuss a mechanism for producing the smooth and continuous line-profile variation shown by the observations. We envision a scenario in which an accretion disk forms abruptly as a result of the tidal disruption of a star by a supermassive black hole, much like what has been discussed above. However, we ascribe the variation of the line profile to a perturbation in the brightness of the disk (a bright spot) which revolves smoothly around the center. A similar scenario has been used to explain the variation of the double-peaked $\mathrm{H} \alpha$ emis- sion line of Arp 102B by Newman et al. (1997). We propose that the revolving bright spot is a precessing warp in the disk, induced by irradiation from the center, and we examine below the qualitative features of this hypothesis.

In a recent work, Pringle (1996) demonstrated that accretion disks irradiated by a central source can become unstable to warping (see also Petterson 1977; Iping \& Petterson 1990). A warped disk is expected to precess. It has been shown that such irradiation-induced warps can occur in X-ray binaries, in the masing disk of NGC 4258, and in "point-symmetric" planetary nebulae (Maloney, Begelman, \& Pringle 1996; Livio \& Pringle 1996; Southwell, Livio, \& Pringle 1997). Here we examine whether an accretion disk in the central engine of NGC 1097 could be subject to this instability, and we estimate the expected precession timescale.

The condition for the instability can be expressed as (Pringle 1996)

$$
\frac{R}{r_{g}} \gtrsim \xi \frac{\eta^{2}}{\epsilon^{2}}
$$

where $R$ is the radial distance in the disk, $r_{g}$ is the gravitational radius defined earlier, $\eta=v_{1} / v_{2}$ is the ratio of the $(R, z)$ and $(R, \phi)$ viscosities, $\epsilon=L / \dot{m} c^{2}$ is the accretion efficiency (with $L$ the accretion luminosity and $\dot{m}$ the mass accretion rate), and $\xi$ is a dimensionless parameter. Analytic estimates give $\xi=16 \pi^{2}$, while numerical results indicate that $\xi \gtrsim 8$. Taking $\eta=1$ and assuming a typical accretion efficiency of $\epsilon \approx 0.1$, we find that the disk can become unstable to warping for $R \sim 800-1600 r_{g}$. This means that the disk in NGC 1097, which would have a radius between $1000 r_{g}$ and $2000 r_{g}$, could become unstable.

The precession timescale of the disk is given by (Pringle 1996)

$$
\tau_{\mathrm{prec}} \approx \frac{12 \pi c \Sigma R^{3} \Omega}{L}
$$

where $\Sigma$ is the surface density and $\Omega$ is the orbital angular velocity. We now proceed to estimate this timescale for the particular case of NGC 1097. We assume the mass of the central black hole to be $M \approx 10^{6} M_{\odot}$. A mass of this order is favored in tidal disruption scenarios for the formation of the disk, since a black hole of this mass would readily disrupt a star before accreting it (Rees 1988; 1990). The $\mathrm{X}$-ray luminosity of the nucleus was found to be $L_{\mathrm{X}} \approx 2$ $\times 10^{41} \mathrm{ergs} \mathrm{s}^{-1}$, from observations with the ROSAT HighResolution Imager (Pérez-Olea \& Colina 1996). The mass of the $\mathrm{H} \alpha$-emitting gas was estimated by Storchi-Bergmann et al. (1995) to be

$$
M_{\mathrm{H} \alpha} \approx 0.24\left(\frac{n}{10^{8} \mathrm{~cm}^{-3}}\right)^{-1}\left(\frac{H_{0}}{75 \mathrm{~km} \mathrm{~s}^{-1} \mathrm{Mpc}^{-1}}\right)^{-2} M_{\odot},
$$

where $n$ is the electron density and $H_{0}$ is the Hubble constant. If the density of the line-emitting gas is in the range expected for a standard broad-line region (i.e., of order $10^{10}$ $\mathrm{cm}^{-3}$ or greater), the observed $\mathrm{H} \alpha / \mathrm{H} \beta$ ratio of about 3 suggests a value of order $10^{12} \mathrm{~cm}^{-3}$ (Rees, Netzer, \& Ferland 1989). Of course, gas with a very low density (on the order of $10^{3} \mathrm{~cm}^{-3}$ ) can also produce an $\mathrm{H} \alpha / \mathrm{H} \beta$ of about 3 , but if this were the case the forbidden lines would also be broad and double-peaked (the Balmer lines are the only 
broad, double-peaked lines observed). Thus the mass of ionized gas in the visible portion of the disk is of order $M_{\text {ring }} \approx 10^{-4} M_{\odot}$. We emphasize that this estimate of the mass refers to the portion of the elliptical ring that emits the $\mathrm{H} \alpha$ line. A larger reservoir of matter (e.g., $10^{-3}$ to $10^{-2} M_{\odot}$ ) may exist exterior to this ring, where the disk is thicker and denser (see, for example, Collin-Souffrin \& Dumont 1990). For parameters typical of NGC 1097, equation (2) therefore gives

$$
\begin{aligned}
\tau_{\text {prec }} \sim & 8.8\left(\frac{M_{\text {ring }}}{10^{-4} M_{\odot}}\right)\left(\frac{M}{10^{6} M_{\odot}}\right)^{1 / 2}\left(\frac{R}{1500 r_{g}}\right)^{-1 / 2} \\
& \times\left(\frac{L}{2 \times 10^{41} \mathrm{ergs} \mathrm{s}^{-1}}\right)^{-1} \mathrm{yr} .
\end{aligned}
$$

This precession timescale is of the right order to explain the observed changes in the profile. A detailed comparison of this model with the observational data would involve detailed modeling of the emission from a warped disk, which is beyond the scope of the present work.

\section{CONCLUSIONS AND SPECULATIONS}

The behavior of the double-peaked Balmer lines of NGC 1097 is so far consistent with the scenario attributing their origin to an accretion disk that has formed abruptly from the tidal disruption of a star by a black hole. There are a number of different phenomena in the disk that can cause it to be nonaxisymmetric and, in turn, result in asymmetries and time variability of the profiles of the lines that it emits. The eccentric precessing disk model discussed above can fit the line profiles quite well, and leads to a reasonable dynamical estimate of the mass of the central black hole. The warped disk model is also plausible (at least qualitatively), since it can reproduce the observed variability timescale. The fact that the emission-line profiles have been varying smoothly and continuously over the past 4 years reinforces our proposal that they originate in a single structure in the accretion flow, such as the accretion disk.

The two disk scenarios that we have discussed above can be used to make predictions (albeit uncertain) about the future evolution of the line profiles. In the case of the irradiation-induced warp, we expect that the pattern of variability that has been observed so far will continue for some time, although eventually (when shadowing starts to play an important role in the disk) the behavior could become chaotic (Livio \& Pringle 1997). This is expected to happen when the inclination angle of some part of the disk changes by more than $90^{\circ}$. In the case of the eccentric pre- cessing disk model, we also expect this pattern of variability to continue, and to be periodic, at least in principle. However, the evolution of the profile shape in this model depends sensitively on the relative timescales for precession and circularization. The latter timescale is expected to be longer than the former (see the estimate of Eracleous et al. 1995), but this question cannot be answered with confidence without detailed numerical calculations.

Whatever the evolution of the profiles of the doublepeaked lines, it is worthy of study. An alternative model that is not discussed in detail here but deserves attention nonetheless is one in which the abrupt appearance of the doublepeaked Balmer lines is the result of the sudden illumination of a preexisting accretion disk by a transient source of ionizing radiation. We speculate that such a transient ionizing source could be associated either with the formation of an elevated structure in the inner accretion disk (e.g., an ionsupported torus; Rees et al. 1982) or with an outburst analogous to a dwarf nova event in the disk. In this context, the variations in the integrated flux of the line would be attributed to variations in the intensity of the ionizing source, and the variations of the line profile could be the result of a bright spot in the disk or a warp induced by the intense illumination. In order to make further progress, the models proposed here should be tested by future observations. The variability of the line profiles is an important aspect of the proposed scenarios and offers a way of testing them by continued spectroscopic monitoring. The phenomenon of the abrupt appearance of double-peaked emission lines in LINERs and LINER-like objects is not confined to NGC 1097 but extends to a few other objects as well (e.g., M81, Pictor A; see $\S 1$ ). NGC 1097 is the most spectacular performer among these objects, and therefore deserves frequent and careful spectroscopic monitoring. We would not like to miss the next episode of this drama!

T. S.-B. acknowledges fruitful discussions with Luc Binette and Charles Bonatto, and partial support from the Brazilian institutions CNPq, CAPES, and FAPERGS. We also thank the referee, R. Antonucci, for useful comments. M. E. acknowledges support from NASA through the Hubble Fellowship grant HF-01068.01-94A awarded by the Space Telescope Science Institute (STScI), which is operated for NASA by the Association of Universities for Research in Astronomy, Inc., under contract NAS 5-26255. M. L. and A. S. W. acknowledge support from NASA grants NAGW-2678 and NAGW-4700, respectively, and A. V. F. acknowledges support from STScI grant AR-05792.01-94A.

\section{REFERENCES}

Antonucci, R. R. J. 1992, in Testing the AGN Paradigm, ed. S. S. Holt,

S. G. Neff, \& C. M. Urry (New York: AIP), 486

Antonucci, R. R. J., Kinney, A. L., \& Ford, H. C. 1989, ApJ, 342, 64

Bartel, N., et al. 1982, ApJ, 262, 556

Bietenholz, M. F., et al. 1996, ApJ, 457, 604

Bower, G. A., Wilson, A. S., Heckman, T. M., \& Richstone, D. O. 1996, AJ, 111,1901

Collin-Souffrin, S., \& Dumont, A. M. 1990, A\&A, 229, 292

Eracleous, M., \& Halpern, J. P. 1994, ApJS, 90, 1 (EH94)

Eracleous, M., Livio, M., Halpern, J. P., \& Storchi-Bergmann, T. 1995, ApJ, 438, 610

Gaskell, C. M. 1983, in Proc. 24th Liège Int. Astrophys. Colloq. (Cointe-

Ougrée: Univ. Liège), 473

Halpern, J. P., \& Eracleous, M. 1994, ApJ, 433, L17

Heckman, T. M. 1980, A\&A, 87, 152

Horne, K., \& Marsh, T. R. 1986, MNRAS, 218, 761

Hummel, E., van der Hulst, J. M., \& Keel, W. C. 1987, A\&A, 172, 32

Iping, R. C., \& Petterson, J. A. 1990, A\&A, 239, 221

Ishisaki, Y., et al. 1996, PASJ, 48, 237

Iyomoto, N., Makishima, K., Fukazawa, Y., Tashiro, M., Ishisaki, Y., Nakai, N., \& Taniguchi, Y. 1996, PASJ, 48, 231

Koratkar, A., Antonucci, R. R. J., Goodrich, R. W., Bushouse, H., \& Kinney, A. L. 1995, ApJ, 450, 501

Koratkar, A., Kinney, A. L., \& Bohlin, R. C. 1992, ApJ, 400, 435

Kormendy, J., et al. 1997, ApJ, 482, L139

Livio, M., \& Pringle, J. E. 1996, ApJ, 465, L55

.1997, ApJ, 486, in press

Maloney, P. R., Begelman, M. C., \& Pringle, J. E. 1996, ApJ, 472, 582

Marsh, T. R. 1988, MNRAS, 231, 1117

Mushotzky, R. F., et al. 1995, MNRAS, 272, P9

Nandra, K., George, I. M., Mushotzky, R. F., Turner, T. J., \& Yaqoob, T. 1997, ApJ, 477, 602

Newman, J. A., Eracleous, M., Filippenko, A. V., \& Halpern, J. P. 1997, ApJ, 485, 570

Pérez-Olea, D. E., \& Colina, L. 1996, ApJ, 468, 191

Petterson, J. A. 1977, ApJ, 216, 827 
Pringle, J. E. 1996, MNRAS, 281, 357

Rees, M. J. 1988, Nature, 333, 523 1990, Science, 247, 817

Rees, M. J., Begelman, M. C., Blandford, R. D., \& Phinney, E. S. 1982, Nature, 295, 17

Rees, M. J., Netzer, H., \& Ferland, G. J. 1989, ApJ, 347, 640

Seaton, M. J. 1979, MNRAS, 187, 73P

Southwell, K. A., Livio, M., \& Pringle, J. E. 1997, ApJ, 478, L29

Storchi-Bergmann, T., Baldwin, J. A., \& Wilson, A. S. 1993, ApJ, 410, L11
Storchi-Bergmann, T., Eracleous, M., Livio, M., Wilson, A. S., Filippenko, A. V., \& Halpern, J. P. 1995, ApJ, 443, 617

Syer, D., \& Clarke, C. J. 1992, MNRAS, 255, 92

Tanaka, Y., et al. 1995, Nature, 375, 659

Wolstencroft, R. D., \& Zealey, W. J. 1975, MNRAS, 173, 51P

Young, P., \& Schneider, D. P. 1980, ApJ, 238, 955

Young, P., Schneider, D. P., \& Shectman, S. A. 1981, ApJ, 245, 1035

Zheng, W., Binette, L., \& Sulentic, J. W. 1990, ApJ, 365, 115 